Solid State Communications, Vol. 9, pp.95-98, 1971. Pergamon Press. Printed in Great Britain.

\title{
MULTIPHONON EFFECTS IN PARAELECTRIC RELAXATIONS
}

\author{
Herbert B. Shore* \\ University of California, San Diego, La Jolla, California \\ and \\ Leonard M. Sander ${ }^{\dagger}$ \\ U:riversity of Michigan, Ann Arbor, Michigan \\ (Received 31 August 1970 by $H$. Suhl)
}

\begin{abstract}
A theory is presented for the temperature dependence of the relaxation rate of paraelectric impurities in alkali-halides. The impurity is regarded as a two-level system coupled linearly to a phonon field. The relaxation rate is evaluated for arbitrary number of phonons, using a Debye spectrum for the lattice. The results agree well with experiments by Kapphan and Luity for relaxation of $\mathrm{OH}^{-}$impurities in $\mathrm{RbBr}$.
\end{abstract}

SUBSTITUTIONAL OH impurities in many alkali-halides can rotate between the six equivalent $\langle 100\rangle$ directions by 'tunneling' through potential barriers.' The six-fold degeneracy among the low lying energy levels will always be partially or completely lifted either by the imposition of an external electric field or by the tunneling itself. ${ }^{2}$ If the populations of the six states are disturbed from thermal equilibrium, relaxation towards equilibrium will occur by means of the interaction of the impurities with the lattice phonons. In a recent experiment, ${ }^{3}$ the relaxation rate $T_{1}^{-1}$ of $\mathrm{RbBr}: \mathrm{OH}$ was measured as a function of temperature $T$. At low temperatures $1 / T_{1}$ is proportional to $T$, a characteristic of one-phonon relaxation. For $T \geqslant 10^{\circ} \mathrm{K}$ the rate increases to approximately $1 / T_{1} \sim A T^{4}$, indicating that multiphonon effects are important in this temperature region.

Dick and Strauch ${ }^{4}$ have attempted to explain

\footnotetext{
* Supported by the National Science Foundation

$\uparrow$ Supported in part by the Horace H. Rachman School of Graduate Studies.
}

these experimental results by using detailed models for the impurity and phonon spectrum and including processes involving one and two phonons. The present authors ${ }^{5}$ have calculated a rate that includes phonon processes to all disorders, using a very primitive model for the phonons : i.e. a linear dispersion relation with no short-wavelength cutoff. The model and the results of reference 5 are similar to a calculation of the relaxation rate for paraelastic defects (such as $\mathrm{O}_{2}^{-}$) by Gosar and Pirc. ${ }^{6}$ In the present communication, we extend the method of reference 5 by including a short wavelength cutoff $\omega_{c}$ (of the order of the Debye frequency) in the phonon spectrum. With this modification, the calculation of $T_{1}^{-1}$ fits the experimental data very well, and illustrates the important features that arise from the strong dipole-lattice coupling.

The major simplification in our model is the replacement of the 6-level $\mathrm{OH}^{-}$system by a two level system. Any rates we calculate with this model will be wrong by a multiplicative constant, but the temperature dependence should be correct if the relaxation of the original system is describable by a single decay time. We use the 
Hamiltonian :

$$
\begin{gathered}
\mathcal{H}=\quad \epsilon S_{z}-\Delta_{0} S_{x}+\sum_{h} \omega_{k} a_{k}^{\dagger} a_{k} \\
i S_{Z} \sum_{k} W_{k}\left(a_{k}-a_{k}^{\dagger}\right) .
\end{gathered}
$$

Here $S_{x}, S_{y} S_{z}$ are spin $1 / 2$ matrices, which describe the two possible orientations of the dipole; $\epsilon$ is the energy difference between the "up" and "down" orientations produced by an external applied electric field, and $\Delta_{0}$ is the 'bare' tunneling matrix element. The third term in $\mathcal{H}$ represents the unperturbed lattice vibrations. The fourth term is the dipole lattice coupling, assumed to be linear in the displacements of lattice atoms. The sign of the inter-action depends on the orientation of the dipole; i.e. the dipole pulls or pushes on its neighbors depending on its orientation.

The Hamiltonian $\mathcal{H}$ is exactly soluble if $\Delta_{0} \quad 0$. This allows one to regard the tunneling as a perturbation and to treat the dipole-lattice coupling exactly. We leave the details to refer ence 5 and give here the results. The relaxation rate is given by:

$$
1 / T, \quad \frac{1}{2} \Delta^{2} \int_{-\infty}^{\infty} \mathrm{d} t \cos \epsilon t[R(t)-1]
$$

where

$$
R(i)=\exp \left\{\sum_{h}\left(W_{k} / \alpha_{k}\right)^{2} \mid\left(n_{h}+1\right) \mathrm{e}^{-\omega_{k} k^{t}}\right.
$$

Here $n_{k}=1 /\left[\exp \left(\beta \omega_{k}\right)-1\right]$ is the Bose distribution function. $\Delta$ is the 'observed' tunneling matrix element, related to $\Delta_{0}$ by

$$
\Delta^{2} \quad \cdots \quad \Delta_{0}^{2} \exp \left\{\sum_{k}\left(H_{k} / \omega_{k}\right)^{2}\left(2 n_{k}+1\right)\right\} .
$$

The temperature dependence of $\Delta$ is an important part of the temperature dependence of $1 / T_{1}$. Equation (2) is valid for $\Delta, 1 / T_{1} \ll \epsilon$. If the exponential in equation (3) is expanded, the term containing $\left(W_{k} / \omega_{k}\right)^{2 n}$ describes all processes involving $n$ phonons.

In order to evaluate equations (3) and (4) we make simple approximations for the $k$ dependence of $W_{k}$ and $\omega_{k}$. The lattice spectrum is taken as $\omega_{k}=v|k|$ up to a cutoff frequency $\omega_{r}$. Since the dipole couples to lattice strains, for long wavelengths $W_{k}$ varies as $|k|^{\frac{k}{2}}$, we then assume that $W_{k}=\alpha_{i}^{1}{ }_{k}^{2}$ for any $k$. With these approximations, one can write

and

$$
\begin{gathered}
R(t) \quad \therefore \exp \left\{3\left(\pi k_{B} T_{0}\right)^{-2}\right. \\
\left.\int_{\omega_{c}} \mathrm{~d} \omega \omega \mathrm{e}^{-i \omega t} /\left(1-\mathrm{e}^{-\beta \omega}\right)\right\}
\end{gathered}
$$

$$
\begin{aligned}
\Delta^{2}(T)= & \Delta_{\omega_{r}}^{2}(0) \cdot \exp \left\{-6\left(\pi k_{B} T_{0}\right)^{-2}\right. \\
& \left.\int_{0}^{\infty} \mathrm{d} \omega \omega /\left(\mathrm{e}^{\beta \omega}-1\right)\right\} .
\end{aligned}
$$

The temperature $T_{0}$ describes the strength of the dipole-lattice coupling; the significance of $T_{0}$ is that multi-phonon effects become important for $T \gtrsim T_{0}$. In terms of the approximation for $W_{k}, T_{0}$ is given by $\left(k_{B} T_{0}\right)^{-2}=\alpha^{2 / 6 a}$, The quantity $\Lambda(0)$ is the observed tunneling matrix element at $T=0$. It is related to $\Lambda_{0}$ by $\Delta^{2}(0) \therefore \Delta_{0}^{2} \exp \left(-\Sigma_{k} W_{k}{ }^{2} / \omega_{k}^{2}\right)$, but can be regarded as an independent parameter. For $k_{B} T \ll \omega_{c}$ the upper limit of the integral in equation (6) can be extended to infinity, with the result that $\Lambda^{2}=\Lambda^{2}(0) \exp \left(-T^{2} / T_{0}^{2}\right)$. (The factor $6, \pi^{2}$ was used in the definition of $T_{0}$ in equations (5) and (6) in order to avoid such factors in calculated quantities such as $\Delta^{2}$.) The renormalization of $\Delta_{0}$ occurs because the lattice is distorted differently for the two orientations of the dipole; the tunneling matrix element $\Delta$ for the lattice-dipole system is reduced from $\Delta_{0}$ by the overlap of the lattice wave functions for the two orientations.

Equations (2), (5) and (6) provide an expression for $1 / T$, in terms of the four adjustable parameters $\epsilon: \Delta(0), T_{0}$ and $\omega_{n}$. To illustrate the dependence of these parameters, we first note that $1 / T$, is independent of $\epsilon$ if $\epsilon \ll k_{B} T$; thus for small external fields $\epsilon$ can be set equal to zero and the factor $\cos \epsilon t$ in equation (2) replaced by unity. The contribution to $1 / T_{1}$ from single phonon processes, obtained by expanding the exponential in equation (5) to first order, is:

$$
\left(1 / T_{1}\right)^{11:} \quad\left(3 \Lambda^{2}(0) / \pi k_{B} T_{0}\right) \cdot\left(T / T_{O}\right) .
$$

The multiphonon contribution can then be written

$$
1 / T_{1}=\left(1 / T_{1}\right)^{11} \cdot F\left(T / T_{0}, \omega_{C} / k_{B} T_{0}\right)
$$

The function $F$ is dimensionless. We have evaluated it numerically by expanding the 
exponential in equation (5) and then evaluating the Fourier transform of equation (2) by a method of repeated convolutions. The integral in equation (6) is easily evaluated as a series. The result is shown in Fig. 1.

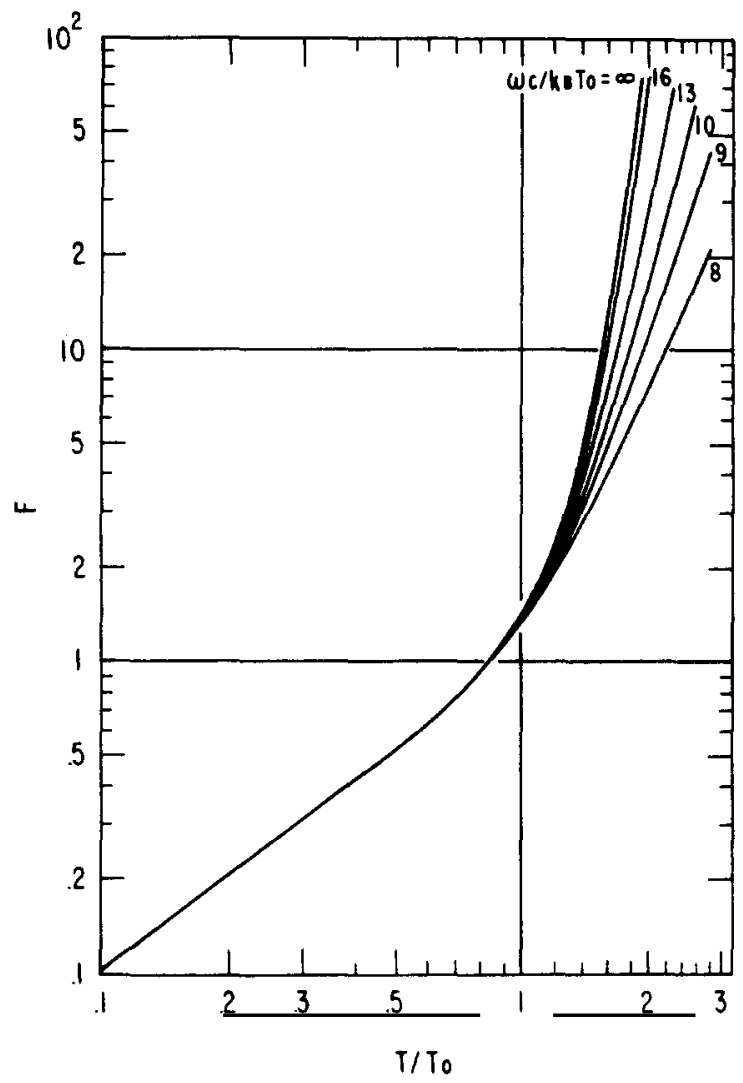

FIG. 1. The function $F$ vs. $T / T_{0}$ for several values of $\omega_{C} / k_{B} T_{0}$.

The most striking feature of the function $F$ is that it depends strongly on $\omega_{C}$ even for $k_{B} T \ll \omega_{c}$. (For example if $T=2 T_{0}$, the relaxation rate for $\omega_{c}=\infty$ is greater than the rate for $\omega_{c}=10 k_{B} T_{0}$ by an order of magnitude!) Thus phonons near the zone boundaries make a major contribution to multiphonon relaxation even for relatively low temperatures. This fact makes it clear that the present calculation, with its simplified phonon spectrum, should be regarded as schematic; the adjustable cutoff is being used here to compensate for the incorrect $k$ dependence of $W_{k}$ and $\omega_{k}$.

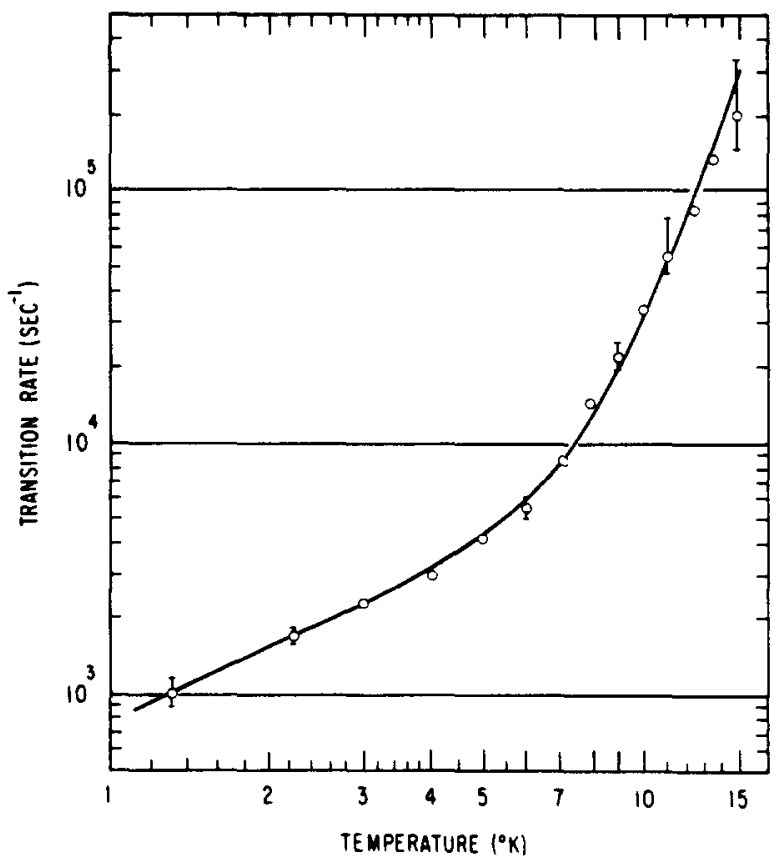

FIG. 2. Relaxation rate of $\mathrm{OH}^{-}$impurities in $\mathrm{RbBr}$ as a function of temperature. The open circles are the data of Kapphan and Luity. The solid line is a theoretical fit using $T_{0}=6.35^{\circ} \mathrm{K}, \omega_{c} / k_{B}=67^{\circ} \mathrm{K}$

The experimental data of Kapphan and Luity for the relaxation of $\mathrm{RbBr}: \mathrm{OH}$ are shown in Fig 2 , along with the best theoretical fit of the form $1 / T_{1}=$ const. $\times F$. The values of the adjustable parameters are $T_{0}=6.35^{\circ} \mathrm{K}, \omega_{c} / k_{B}=$ $67^{\circ} \mathrm{K}$. The apparent $T^{4}$ behavior of $1 / T_{1}$ for $T \gtrless 7^{\circ} \mathrm{K}$ is, we believe, of no significance; in fact a curve of the form $1 / T_{1}=\mathrm{AT}+\mathrm{BT}^{4}$ does not give a good fit to the data. The low value of $\omega_{c}$ is somewhat disturbing; however the Debye temperature of $\mathrm{RbBr}$ is also rather low $\left(\theta_{D}=130^{\circ} \mathrm{K}\right.$ at $T=50^{\circ} \mathrm{K}$, so that this value of $\omega_{c}$ is not too unreasonable.

In summary the calculation described above illustrates three major points: the importance of including multiphonon processes to all orders for $T \gtrless T_{0}$, the temperature dependence of the renormalization of $\Delta_{0}$, and the contribution of phonons neat the zone boundary to high-order processes. The present calculation is much too crude to yield reliable information about the nature of the dipole-lattice coupling in paraelectrics. However, we believe that such 
information could be gained from a careful study of the temperature dependence of the relaxation. In particular, a multiphonon calculation using a realsitic spectrum would be extremely enlightening.
Acknowledgements - We would like to thank B.G. Dick and F. Lüty for valuable comments.

\section{REFERENCES}

1. LÜTY F., J. Phys. C4, suppl. 8-9 120 (1967). ESTLE T.L., Phys. Rev. 176, 1056 (1968). NARAYANAMURTI V. and POHL R.O., Rev. mod. Phys, 42, 201 (1970).

2. SAUER P., SCHIRMER O. and SCHNEIDER J., Phys. Status. Solidi. 16, 79 (1965). SHORE H.B., Phys. Rev. 151, 570 (1966).

3. KAPPHAN S. and LÜTY F., Solid State Commun. 8, 349 (1970).

4. DICK B.G. and STRAUCH D. (to be published).

5. SANDER L.M. and SHORE H.B. (to be published).

6. GOSAR P. and PIRC R., Proc. XIV-th Colloque Ampere, Ljubljana, p. 636, 1966, North-Holland Amsterdam, (1967). PIRC R. and GOSAR P., Phys, kondens. Materie, 9, 377 (1969).

7. SHORE H:B., Phys. Rev. Lett. 17, 1142 (1966). Wir entwickeln eine Theorie für die Temperaturabhängigkeit der
Relaxations-rate paraelektrischer Verunreinigungen in Alkalihalogeniden.
Dabei wird die Verunreinigung als ein Zweiniveausystem betrachtet,
das linear an ein Phononenfeld angekoppelt ist. Wir berechnen die
Relaxationsrate für eine beliebige Anzahl von Phononen unter Benutzung
eines Debyespektrums für das Gitter. Die Resultate stimmen mit
Experimenten von Kapphan and Lüty für die Relaxation von OH
Verunreinigungen in RbBr gut überein. 